\title{
A utilização do forjo na captura do caranguejo uçá (Ucides cordatus) na comunidade de Tapebas em Fortaleza - CE
}

\author{
Herbster Ranielle Lira de Carvalho ${ }^{1}$ \\ Marco Antonio Igarashi ${ }^{2 *}$ \\ ${ }^{1}$ Centro de Tecnologia em Aqüicultura do Departamento de Engenharia de Pesca \\ Universidade Federal do Ceará, Fortaleza, Brasil \\ ${ }^{2}$ Departamento de Engenharia de Pesca da UFC \\ Campus Universitário do Pici, Bloco 827 \\ Caixa Postal 12.168, CEP 60455-760, Fortaleza - CE, Brasil \\ *Autor para correspondência \\ igarashi@ufc.br
}

Submetido em 28/05/2008

Aceito para publicação em 21/10/2008

\section{Resumo}

O presente trabalho foi realizado no Estuário do Rio Ceará situado no município de Caucaia - CE, de agosto a dezembro de 2003, com o principal objetivo de contribuir com as informações sobre a utilização do "forjo" equipamento de captura. A captura de caranguejo uçá Ucides cordatus (Linnaeus, 1763) na comunidade de Tapebas, Estado do Ceará, é principalmente feito pelo método do "forjo". O "forjo" é uma armadilha confeccionada com lata, câmara de ar de pneu, arame e madeira do mangue. Cada pescador usa de 80 a 100 "forjos" colocados em várias áreas no mangue. Em nosso estudo, o comprimento médio dos 2.100 caranguejos machos e fêmeas capturados foram de $45,54 \mathrm{~mm}$ e de $44,78 \mathrm{~mm}$, respectivamente. A produção obtida pelos caranguejeiros nas áreas de manguezais do Rio Ceará é comercializada próximo à comunidade. Neste contexto, a produção de caranguejo na comunidade de Tapebas é importante onde há poucas alternativas de renda.

Unitermos: catador de caranguejo, forjo, Ucides cordatus, Manguezal, seletividade

\section{Abstract}

Use of the "forjo" in capturing uça crabs (Ucides cordatus) in the Tapebas community, Fortaleza CE. The present work was carried out in the estuary of the Ceará river situated in the municipality of Caucaia - CE, from August to December 2003, with the aim of contributing to information about utilization of the "forjo" method of trapping. The capture of the crab Ucides cordatus (Linnaeus, 1763) in the Tapebas community, Ceará State, is mainly done by "forjo". The "forjo" is a crab trap made out of an oil can, a tyre innertube, wire, and mangrove sticks. Each crab gatherer uses from 80 to 100 "forjos", placed in various areas of the mangrove. In our study, the average lengths of 2001 captured males and females were $45.54 \mathrm{~mm}$ and $44.78 \mathrm{~mm}$, respectively. The crabs captured from the mangrove area of the Ceará river are marketed within the vicinity of the community itself, and the crab production in the Tapebas community is extremely important on account of the fact that there are few other alternatives for income.

Key words: crab gatherers, forjo, Ucides cordatus, Mangrove, selectivity 


\section{Introdução}

No Brasil, a captura do caranguejo-uçá, Ucides cordatus (L., 1763) é uma das atividades mais antigas de extrativismo (Geo Brasil, 2002; Passos e Di Beneditto, 2005). Este crustáceo é um importante recurso econômico e de subsistência em todas as áreas de manguezal no Brasil, do Amapá a Santa Catarina (Nordi, 1992; Maneschy, 1996; Costa-Neto e Lima, 2000; Ivo et al., 2000; Paiva-Sobrinho e Alves, 2000; Wolff et al., 2000; Barros, 2001; Alves, 2002; Fiscarelli e Pinheiro, 2002; Graser e Diele, 2004; Souto, 2004) limite de ocorrência desse ecossistema no litoral Atlântico da América do Sul (Coelho-Júnior, 2000).

O U. cordatus é um crustáceo que habita as regiões de mangue nas zonas entremarés, em galerias (tocas) escavadas no lodo, que podem atingir $1,60 \mathrm{~m}$ de profundidade; alimentam-se de vegetais e de matéria orgânica em decomposição (Paiva et al., 1971; Nascimento, 1993). Essa espécie é territorialista, os maiores indivíduos são encontrados no mesolitoral, enquanto os menores predominam nas zonas mais altas do mangue (Ostrensky, 2001).

A atividade de catação (captura) é realizada principalmente na região Nordeste (Alves e Nishida, 2002). A espécie é artesanalmente explorada (Nordi, 1994a e b; Botelho et al., 2000; Nóbrega e Nishida, 2003; Glaser e Diele, 2004; Alves et al., 2005; Diele et al., 2005) e anualmente a captura pode chegar a $7 \mathrm{t}$ por $\mathrm{km}^{2}$ de vegetação de mangue (Araújo, 2006).

A população da Comunidade de Tapebas, localizada às margens do rio Ceará (Caucaia-CE), tem como principal atividade econômica a captura do U. cordatus. Segundo algumas informações dos nativos da comunidade de Tapebas, o U.cordatus foi até meados de 1980, capturado pelo método do braceamento, onde o catador introduzia o braço na toca do animal até capturá-lo.

Porém, começaram a surgir outros apetrechos facilitando a captura do U. Cordatus, o que levou os catadores a procurar novas técnicas de captura, como a utilização do "forjo".

Até o presente, há uma carência de informações a respeito da utilização desse petrecho na captura do
U. Cordatus. Nesse sentido, o objetivo da pesquisa foi diagnosticar a sua utilização pela comunidade de Tapeba.

\section{Materiais e Métodos}

A presente pesquisa foi realizada de agosto a dezembro de 2003 com periodicidade semanal, no estuário do rio Ceará, litoral norte do Estado do Ceará. O local de estudo situa-se entre as coordenadas geográficas 380 24' e 390 00' de longitude Oeste e 30 11' e 3023' de latitude Sul.

Foram realizadas visitas semanais aos catadores quando foi efetuada a biometria de 2.100 espécimes de $U$. cordatus capturados e trazidos à comunidade. Durante a biometria dos indivíduos, foi medido o comprimento das carapaças (tomado no plano de simetria e sobre o dorso do corpo, estendendo-se da margem anterior da fronte à margem posterior da carapaça) (Ivo e Vasconcelos, 2000).

A distribuição de freqüência de comprimento de carapaça por classe; comprimento médio de carapaça por classe, freqüência simples, relativa e acumulada, de machos e fêmeas de U. cordatus (Linnaeus, 1763) capturados com o "forjo" foram analisados utilizando um paquímetro de $200 \mathrm{~mm}$ (precisão de $0,1 \mathrm{~mm}$ ). As informações sobre a construção e a armação do "forjo" foram obtidas da observação direta da atividade de captura.

\section{Resultados e Discussão}

Além do forjo e braceamento, existem outras formas de captura de caranguejo como o uso da redinha, carbureto, vanga (ou cavadeira) (Pinheiro e Fiscarelli, 2001), ratoeira e raminho (Ivo e Gesteira, 1999).

O "forjo" é construído com lata de óleo cilíndrica (Figura 1). No interior da lata, prende-se a isca. $\mathrm{O}$ caranguejo penetra na lata atraída pela isca.

Cada catador sai de casa pela manhã levando consigo de 80 a 100 armadilhas, "forjos" (média 90 armadilhas). As armadilhas são confeccionadas pelos próprios pescadores que compram parte do material 


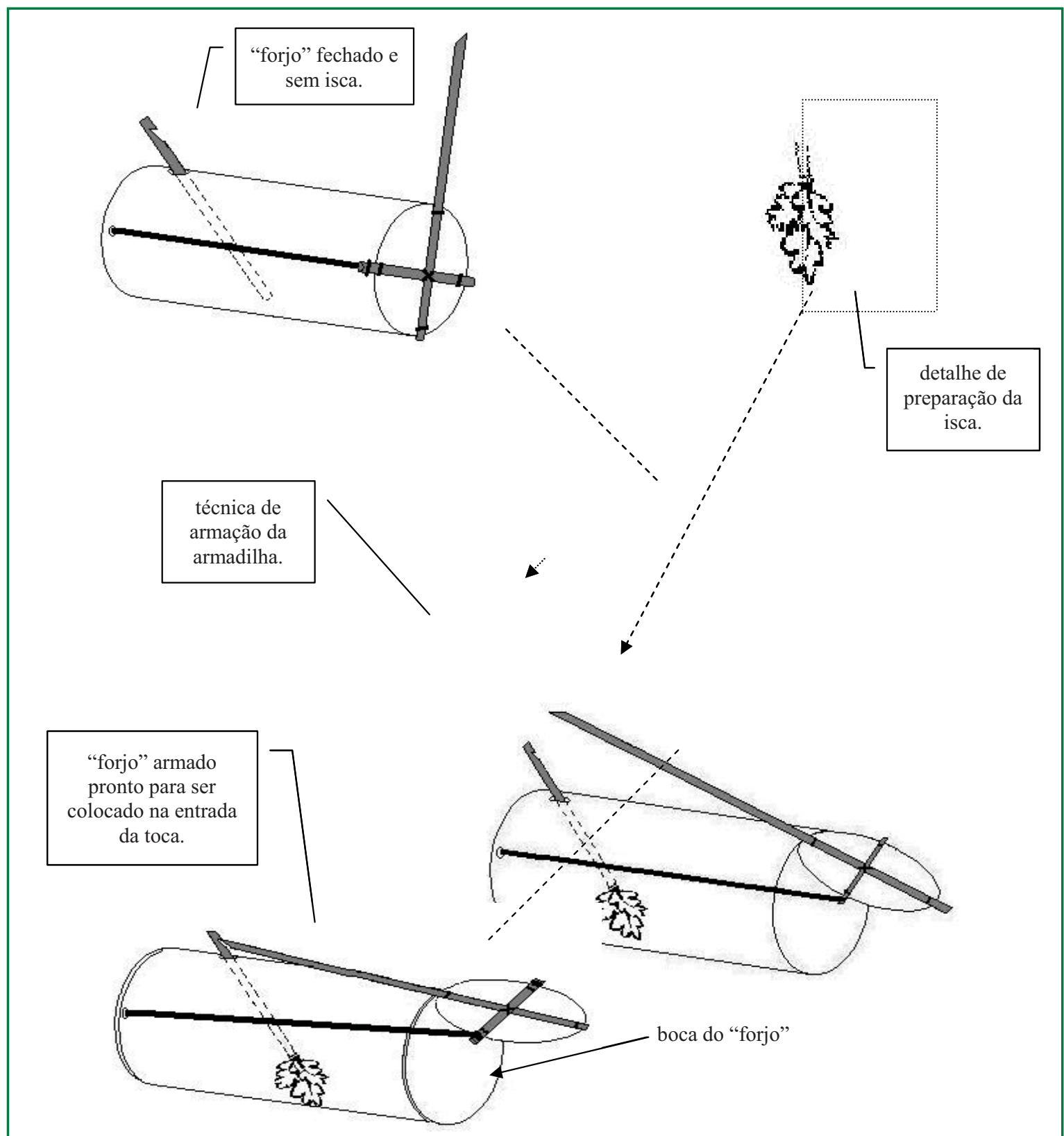

FIGURA 1: Preparação do "forjo" com isca (folha do mangue).

(latas, câmaras de ar de pneu e arame) e outra parte retiram do mangue (madeira para reforço e confecção do sistema de disparo da armadilha).

As armadilhas são armadas de forma a fechar totalmente a entrada das tocas dos caranguejos e folhas retiradas do mangue-sapateiro (Rhizophora mangle) são usadas como iscas. Em seguida, é fechado o suspiro da toca, garantindo que o animal não tenha por onde sair.

O trabalho dos catadores segue o seguinte cronograma: de segunda a quinta-feira colocam as armadilhas pela manhã e as retiram à tarde. Todavia, os animais capturados durante a semana são estocados vivos em tachos feitos de pneu. Na sexta-feira, os animais são agrupados em cordas com dez animais e são vendidos direto ao consumidor em pequenas barracas de palha montadas às margens da rodovia, que liga Fortaleza a Caucaia-CE. Alguns catadores vendem aos atravessadores e outros direto aos donos de barracas de praia, e tem ainda aqueles pescadores que levam os animais para vender em pontos próximos ao mercado São Sebastião (Fortaleza - CE). 
O U. cordatus tem vida relativamente longa, com baixa taxa de crescimento e de predação natural, tendo em vista o restrito número de níveis tróficos da cadeia alimentar dos manguezais (Paiva, 1997). No entanto, a captura do $U$. cordatus é intensa, tendendo a tornar economicamente inviável a manutenção da espécie, devido ao elevado nível de captura a que está sendo submetido este recurso pesqueiro.

A obtenção de dados a partir de captura comercial demonstra que o "forjo", captura indivíduos de vários tamanhos. Durante o período de estudo, dos 2100 espécimes de $U$. cordatus capturados com o "forjo" pela comunidade Tapeba; $76,7 \%$ foram machos.

Foi observado que o comprimento médio de captura foi de $45,54 \mathrm{~mm}$ para machos e de $44,78 \mathrm{~mm}$ para fêmeas (Tabela 1). O U. cordatus tem seu comprimento, estabilizado entorno de $68,0 \mathrm{~mm}$ para fêmeas e $73,0 \mathrm{~mm}$ para machos, com cinco anos de idade aproximadamente (Paiva, 1973).

O uso do "forjo" na captura do U. cordatus demonstrou capturar fêmeas de tamanho pequeno e conseqüentemente, baixa fecundidade. Segundo Pinheiro e Fiscarelli (2001), se considerarmos a maturidade funcional como o tamanho com que os animais se encontram maturos fisiológica e morfologicamente para a reprodução, então o tamanho mínimo de captura seria estabelecido em $52 \mathrm{~mm}$ para os machos e $43 \mathrm{~mm}$ para as fêmeas. De acordo com os mesmos autores, as fêmeas ovígeras de $U$. cordatus apresentaram tamanho variando entre 37 e $73 \mathrm{~mm}$. Segundo Mota Alves (1975), a referida espécie pode atingir sua primeira maturação sexual com comprimento médio de $35,4 \mathrm{~mm}$ para machos e $37,4 \mathrm{~mm}$ para fêmeas. Além disso, o U. cordatus se reproduz uma vez por ano após atingir a primeira maturação sexual (Paiva, 1997).

Segundo Ostrensky (2001), o tamanho comercial do caranguejo é atingido geralmente após o quinto ano de vida. Segundo Diele (2000), U. cordatus demora cerca de oito anos para atingir um tamanho de 60 $\mathrm{mm}$ nos manguezais do Rio Caeté (PA). De acordo com Nascimento (1993), para o U. cordatus alcançar um bom tamanho comercial, demoraria uns 10 anos. Portanto, vale ressaltar que os caranguejos que são comercializados em Fortaleza possuem vários anos de idade podendo ter de 6 a 10 anos.

TABELA 1: Distribuição de freqüência de comprimento de carapaça por classe; comprimento médio de carapaça por classe, freqüência simples, relativa e acumulada, de machos e fêmeas de Ucides cordatus (Linnaeus, 1763) capturados com o "forjo".

\begin{tabular}{ccccccc}
\hline \multirow{2}{*}{$\begin{array}{c}\text { Classes de compri- } \\
\text { mento de carapaça } \\
(\mathbf{m m})\end{array}$} & \multicolumn{2}{c}{$\begin{array}{c}\text { Freqüiência simples } \\
\text { (n⿳⺈ de indivíduos) }\end{array}$} & \multicolumn{2}{c}{ Freqüência relativa (\%) } & \multicolumn{2}{c}{$\begin{array}{c}\text { Freqüência relativa } \\
\text { acumulada (\%) }\end{array}$} \\
\cline { 2 - 7 } & Macho & Fêmea & Macho & Fêmea & Macho & Fêmea \\
\hline $38,0 \mid-40,0$ & 367 & 123 & 22,8 & 25,0 & 22,8 & 25,0 \\
$40,0 \mid-42,0$ & 166 & 55 & 10,3 & 11,1 & 33,1 & 36,1 \\
$42,0 \mid-44,0$ & 183 & 60 & 11,3 & 12,2 & 44,5 & 48,3 \\
$44,0 \mid-46,0$ & 186 & 62 & 11,5 & 12,6 & 56,0 & 60,9 \\
$46,0 \mid-48,0$ & 198 & 65 & 12,3 & 13,2 & 68,4 & 74,1 \\
$48,0 \mid-50,0$ & 148 & 50 & 9,2 & 10,1 & 77,6 & 84,3 \\
$50,0 \mid-52,0$ & 120 & 42 & 7,4 & 8,5 & 85,0 & 92,8 \\
$52,0 \mid-54,0$ & 100 & 35 & 6,2 & 7,1 & 91,2 & 100,0 \\
$54,0 \mid-56,0$ & 68 & - & 4,2 & - & 95,5 & - \\
$56,0 \mid-58,0$ & 48 & - & 3,0 & - & 98,5 & - \\
$58,0 \mid-60,0$ & 24 & - & 1,5 & - & 100,0 & - \\
\hline
\end{tabular}


A comunidade Tapeba é muito carente e necessita com urgência de uma maior atenção das autoridades. A fim de minimizar a intensa atividade de captura do $U$. cordatus, que atualmente é sua principal fonte de renda.

\section{Agradecimentos}

Agradeço imensamente ao Pofessor Yoshiaki Deguchi (Nihon University, Japan) pelas importantes informações sobre caranguejos e dois revisores anônimos pelas valiosas sugestões ao manuscrito.

\section{Referências}

Alves, R. R. da N. 2002 Estrutura populacional de Ucides cordatus cordatus (L. 1763) (Decapoda, Brachyura) e a atividade de coleta no mangue do estuário do rio Mamanguape, Paraíba: Um enfoque social e etnoecológico. Dissertação de Mestrado, Universidade Federal da Paraíba, Brasil, 139pp.

Alves, R. R. N.; Nishida, A. K. A. 2002. Ecdise do caranguejo-uçá, ucides cordatus 1 . (decapoda, brachyura) na visão dos caranguejeiros. Interciencia, 27 (3): 110-117.

Alves, R. R. N.; Nishida, A. K.; Hernández, M. I. M. 2005. Environmental perception of gatherers of the crab "caranguejo-uçá" (Ucides cordatus, Decapoda, Brachyura) affecting their collection attitudes. Journal Ethnobioogy Ethnomedic, 1 (10): Disponível $\mathrm{em}<\mathrm{http} / /$ www.ethnobiomed. com/ content $/ 1 / 1 / 10>$. Acesso em 10 de novembro de 2007.

Araújo, A. R. 2006. Fishery statistics and commercialization of the mangrove crab, Ucides cordatus (L.), in Bragança - Pará Brazil. PhD Thesis, University of Bremen, Germany, 176pp.

Barros, C. M. B. 2001. Conhecimento tradicional, conservação e sobrevivência: A importância da participação dos caranguejeiros na definição de políticas de proteção e manejo do manguezal de Mucuri - Bahia. Dissertação de Mestrado, Universidade Federal de Alagoas, Brasil, 149pp.

Botelho, E. R.; Santos, M. C. F.; Pontes, A. C. P. 2000. Algumas considerações sobre o uso da redinha na captura do caranguejo ucá, Ucides cordatus (Linnaeus, 1763), no litoral sul de PernambucoBrasil. Boletim Técnico e Científico do CEPENE, 8 (1): 55-71.

Coelho Junior, C. 2000. Impactos da carcinicultura sobre os estuários e o ecossistema Manguezal. Anais do Seminário Internacional, Perspectivas e Implicações da Carcinicultura Estuárina de Estado de Pernambuco, Recife, Brasil, p.58-73.

Costa-Neto, E. M.; Lima, K. L. G. 2000 Contribuição ao estudo da interação entre pescadores e caranguejos: Considerações etnobiológicas em uma comunidade pesqueira. Actualidades Biológicas, 22 (73): 195-202.

Diele, K. 2000. Life history and population structure of the exploited mangrove crab U. cordatus (L.) (Decapoda: Brachyura) in the Caeté estuary, North Brazil. PhD Thesis, Universität Bremen, Germany, 103pp.
Diele, K.; Koch, V.; Saint-Paul, U. 2005. Population structure, catch composition and CPUE of the artisanally harvested mangrove crab Ucides cordatus: Indications for overfishing? Aquatic Living Resourse, 18 (2): 169-178.

Fiscarelli, A. G.; Pinheiro, M. A. A. 2002. Perfil sócio-econômico e conhecimento etnobiológico do catador de caranguejo-uçá, Ucides cordatus (Linnaeus, 1763), nos manguezais de Iguape (24\% $41^{\prime} \mathrm{S}$ ), SP, Brasil. Actua Biology, 24 (77): 129-142.

Geo Brasil. 2002. Perspectivas do Meio Ambiente. $1^{\text {a }}$ ed. Instituto Brasileiro do Meio Ambiente e dos Recursos Naturais e Renováveis, Brasília, Brasil, 447pp.

Graser, M.; Diele, K. 2004 Asymmetric outcomes: Assessing central aspect of biological, economical and social sustainability of a mangrove crab fishery, Ucides cordatus (Ocypodidae), in North Brazil. Ecological Economics, 49: 361-373.

Ivo, C. T. C.; Dias, A. F.; Botelho, E. R. de O.; Mota, R. I.; Vasconcelos, J. A. de; Vasconcelos, E. M. S. de 2000 Caracterização das populações de caranguejo-uçá, Ucides cordatus cordatus (Linnaeus, 1763), capturadas em Estuários do Nordeste do Brasil. Boletim Técnico Científico do CEPENE, 8 (1): 9-43.

Ivo, C. T. C.; Gesteira, T. C. V. 1999. Sinopse das observações sobre a bioecologia e pesca do caranguejo-uçá, Ucides cordatus cordatus (Linnaeus, 1763), capturado em estuários de sua área de ocorrência no Brasil. Boletim Técnico Científico do CEPENE, 7 (1): 9-52.

Ivo, C. T. C.; Vasconcelos, E. M. S. 2000. Potencial reprodutivo do caranguejo-uçá, Ucides cordatus cordatus (Linnaeus, 1763), capturado na região estuarina do rio Curimatau, (Canguaretama, Rio Grande do Norte, Brasil). Boletim Técnico Científico do CEPENE, 8 (1): 7-14.

Maneschy, M. C. 1996 Pescadores nos manguezais: estratégias, técnicas e relações sociais de produção na captura do caranguejo. In: Furtado, W. L. \& Mello, A. F. (Eds). Povos das águas: Realidade e perspectivas na Amazônia. MCT/CNPq/MPEG, Belém, Brasil, 292pp.

Mota Alves, M. I. 1975. Sobre a reprodução do caranguejo-uçá, Ucides cordatus (Linnaeus, 1763), em mangues do Estado do Ceará (Brasil). Arquivo de Ciências do Mar, 15 (2): 84-91.

Nascimento, S. A. 1993. Biologia do caranguejo-uçá Ucides cordatus (Linnaeus, 1763). ADEMA, Aracajú, Brasil, 48pp.

Nóbrega, R. R.; Nishida, A. K. 2003. Aspectos socioeconômicos e percepção ambiental dos catadores de caranguejo-uçá Ucides cordatus cordatus (L. 1763) (Decapoda, Brachyura) do estuário do rio Mamanguape, Nordeste do Brasil. Interciencia, 28: 36-43.

Nordi, N. 1992 Os catadores de caranguejo-uçá (Ucides cordatus) da região de Várzea Nova (PB):uma abordagem ecológica e social. São Carlos. Tese de Doutorado, Universidade Federal de São Carlos, Brasil, 107pp.

Nordi, N., 1994a. A produção dos catadores de caranguejo-uçá (Ucides cordatus) na região de Várzea Nova, Paraíba, Revista Nordestina de Biologia, 9 (1): 71-77.

Nordi, N. 1994b A captura do caranguejo-uçá (Ucides cordatus) durante o evento reprodutivo da espécie: o ponto de vista dos caranguejeiros. Revista Nordestina de Biologia, 9 (1): 41-47.

Ostrensky, A . 2001. Ucides cordatus. Ecologia e Desenvolvimento, 98: 33 .

Paiva, M. P. 1973. Recursos pesqueiros e a pesca na bacia do Rio Parnaíba (Brasil). Boletim Cearense de Agronomia, 14 (1): 49-82. 
Paiva, M. P. 1997. Recursos pesqueiros estuarinos e marinhos do Brasil. EUFC, Fortaleza, 1997. 278 pp.

Paiva, M. P.; Bezerra, R. C. F.; Fonteles-Filho, A. A. 1971. Tentativa de avaliação dos recursos pesqueiros do Nordeste brasileiro. Arquivo de Ciências do Mar, 11 (1): 1-43.

Paiva-Sobrinho, R.; Alves, E. J. 2000 Avaliação preliminar do impacto da coleta do caranguejo-uçá (Ucides cordatus), na densidade da espécie em alguns manguezais dos municípios de Ilha Comprida e Cananéia, SP. Anais da $\mathbf{1 3}^{\mathrm{a}}$ Semana Nacional de Oceanografia, Itajaí, Brasil, p.486-488.

Passos, C. A.; Di Beneditto, A. P. M. 2005. Captura comercial do caranguejo-uçá, Ucides cordatus (L., 1763), noManguezal de Gargaú, RJ. Biotemas, 18 (1): 223-231.
Pinheiro, M. A. A.; Fiscarelli, A. G. 2001. Manual de apoio à fiscalização do caranguejo-uçá (Ucides cordatus). UNESP/CEPSUL/IBAMA, Jaboticabal, Brasil, 43pp.

Souto, F. J. 2004. Da lama ao caos: A ciência que veio do mangue. Tese de Doutorado, Universidade Federal de São Carlos, Brasil, 505pp.

Wolff, M.; Koch, V.; Isaac, V. 2000 A trophic flow model of the Caeté Mangrove Estuary (North Brazil) with considerations for the sustainable use of its resources. Estuarine, Coastal and Shelf Science, 50 (6): 789-803. 